\title{
¿Equidad de género en la enseñanza de las Matemáticas?
}

\section{Gender Equality in Mathematics Education?}

\author{
Ronny Gamboa Araya ${ }^{1}$ \\ Escuela de Matemática \\ Universidad Nacional \\ Heredia, Costa Rica \\ rgamboa@una.ac.cr
}

Recibido 02 de setiembre de 2011 • Aceptado 23 de febrero de 2012

\begin{abstract}
Resumen. La enseñanza de las Matemáticas se ha visto influenciada por la presencia de ciertos estereotipos que han determinado el comportamiento y afectado el rendimiento del estudiantado en dicha disciplina. Diversas investigaciones han señalado la relación de inequidad que hombres y mujeres poseen respecto a las matemáticas y la responsabilidad que el profesorado tiene en su construcción. Por tal motivo se plantea la necesidad de llevar a cabo acciones concretas que permitan la construcción de relaciones de equidad en dicha disciplina, basadas en los principios de respeto, solidaridad y ayuda mutua entre los géneros.
\end{abstract}

Palabras claves. Enseñanza, matemática, hombres, mujeres, género, desigualdad, equidad.

Abstract. Mathematics education has been influenced by certain stereotypes, which have shaped the behavior of students and have affected their performance in this discipline. Research has pointed out relationships of inequality between men and women in the field of Mathematics and it has exposed the responsibility of teachers on the formation of such relationships. For that reason, it is necessary to undertake specific actions to promote gender equality in this discipline, based on the principles of respect, solidarity and mutual support between genders.

Keywords. Education, Mathematics, men, women, gender, inequality, equality.

\section{Introducción}

La igualdad de oportunidad educativa ha sido un importante principio del pensamiento democrático y de la justicia solidaria en las sociedades capitalistas (Cervini, 2002). El autor señala que el avance en técnicas de medición de los logros educativos y otros conceptos conexos, como el coeficiente intelectual y la aptitud, y el surgimiento de metodologías para el análisis de sus

Máster en Matemática Educativa por el Centro de Investigación y Estudios Avanzados del Instituto Politécnico Nacional, México. Licenciado en la Enseñanza de la Matemática por la Universidad Nacional. Se ha desempeñado como director, investigador y académico de la Escuela de Matemática de la Universidad Nacional. 
determinantes han dado un importante impulso al desarrollo de investigaciones que han mostrado la existencia de disparidades sociales en los logros educativos.

Género es una categoría que permite analizar las relaciones sociales entre hombres y mujeres. Por género se entiende la construcción social de los sentidos que para las sociedades tiene el ser hombre o mujer, significados que condicionan la actuación, valoración y distribución del poder (real y simbólico) inequitativo para las mujeres como grupo (...). (González, 2003, pp. 132-133)

González-Pienda et al. (2006) indican que en la actualidad se continúa investigando sobre las diferencias en las actitudes de mujeres y hombres, así como sobre las causas de tales diferencias. En las dos últimas décadas ha existido un notable crecimiento en los estudios de género que analizan la situación de las mujeres en el área de las matemáticas, por lo que es posible delimitar dos grandes interrogantes en esta línea de investigación: ¿Existe diferencias entre hombres y mujeres en su actuación en matemáticas? Si esto fuera cierto, ¿qué factores (culturales, educativos, biológicos, psicológicos e instrumentales) influyen en las diferencias? (González, 2003).

Al respecto, Toríz (2004) indica que el tema de relaciones de género y matemáticas ha sido de gran interés entre la comunidad académica y ha generado una amplia bibliografía sobre los patrones de desempeño de hombres y mujeres en matemáticas; destaca, además, que la mayoría de dichas investigaciones se han desarrollado en países como Inglaterra, Estados Unidos y Australia, mientras que en los países de habla hispana apenas ha empezado a discutirse.

El propósito del presente documento es mostrar aspectos relacionados con género y matemáticas, resultados de algunas investigaciones en dicha área y algunas propuestas orientadas al desarrollo de una educación de equidad, con el objetivo de evidenciar la relación de desigualdad que se presenta en la enseñanza de las matemáticas y de crear conciencia sobre la necesidad de realizar un cambio orientado por el principio de igualdad.

\section{Género y Matemáticas}

La presencia profesional de los hombres tradicionalmente ha "dominado" ciertas áreas como las matemáticas, la física y la tecnología (Olaz, 1997; Postigo, Pérez y Sanz, 1999; Salomone, 2007). En el caso de las matemáticas, históricamente se ha observado que la mayoría de las personas dedicadas al desarrollo de esta disciplina son hombres, lo que sugiere que a las mujeres se les ha "imposibilitado" su acceso al conocimiento matemático. A pesar de ello, han existido mujeres matemáticas que no se suelen mencionar en los libros de texto y, si se hace en alguna ocasión, estas son citadas por su apellido, por lo que es frecuente que se suponga que es un hombre (Jimeno, 2002).

Desde que la matemática empezó a profesionalizarse, alrededor del siglo XVIII, se puso en tela de juicio la capacidad de las mujeres para dedicarse a ella. Se estimó conveniente educar a las mujeres, pero no instruirlas en materias consideradas "poco femeninas". Puesto que su formación y dedicación fue tan distinta a la de los hombres, su producción matemática no gozó de igual resonancia, y las mujeres han quedado excluidas de la mayoría de textos de historia de la matemática escritos por hombres (...) (Bayer, 2004, p. 55). 
Al respecto, Espinosa (2010) apunta que en las sociedades occidentales modernas, las matemáticas se consideran un dominio masculino, y las mujeres que deciden entrar en este espacio son catalogadas como aburridas, feas o que están en busca de esposo. Las pocas mujeres con acceso a la educación y que se dedicaron a las matemáticas solían trabajar de forma anónima bajo la protección de sus padres, maridos o colegas masculinos; pero sin otorgárseles los reconocimientos correspondientes. Las mujeres matemáticas de "hoy" no se resignan al anonimato ni a trabajar a la sombra de sus colegas masculinos (Bayer, 2004).

Jimeno (2002) señala que desde la década de los años setenta los temas relacionados con las diferencias entre las mujeres y los hombres, y sus logros en matemáticas, así como la menor presencia de ellas en carreras universitarias relacionadas con este ámbito de conocimiento han sido temas de investigación.

La ciencia se ha estructurado desde un modelo masculino que ha causado que a las mujeres se les haya excluido de esta, lo que trajo como consecuencia una "generalización" de la idea de que ellas no son aptas para el conocimiento científico. Esto se ha utilizado como "evidencia" para explicar la menor tendencia de estas hacia las matemáticas, explicada a veces por razones biológicas, otras veces por razones ambientales o el por el miedo a perder admiradores si se dedican a estudios "masculinos" (Susi, 1994).

En este sentido, Postigo et al. (1999) indican que “(...) parece razonable que aquellas estudiantes que no creen tener la suficiente competencia para enfrentarse a disciplinas en las que tradicionalmente se considera que la capacidad intelectual juega un papel mayor que en otras materias (por ejemplo, matemáticas y ciencias) opten, en el sistema educativo, por opciones en las que no tienen que poner a prueba, desde su propio punto de vista esta habilidad (por ejemplo, filosofía y letras, historia, filología)" (p. 256).

Caprile, Vallès, Potrony, Crèixams y Arasanz (2008) señalan que distintas investigaciones han reflejado la existencia de estereotipos respecto a mujeres y hombres en matemáticas, por ejemplo, que las mujeres son menos hábiles que los hombres en esta disciplina y más hábiles en lenguas, o que las mujeres tienen más dificultad que los hombres para la orientación espacial y más facilidad para las relaciones sociales y la comunicación en general.

Dentro de esta perspectiva de desigualdad de género respecto a las Matemáticas, la estructura del entorno educativo también ha jugado un papel destacable. Subirats (1994) señala que en el currículo escolar se ha juzgado como importantes para la "vida" materias como matemáticas, historia o lenguaje y se han menospreciado actividades como preparar comida, cuidar un niño, entre otras, casi todas relacionadas típicamente con las mujeres.

El papel docente en el establecimiento de estas relaciones de género y matemáticas no se puede negar. Jimeno (2002) señala que las investigaciones apuntan que aunque no se puede afirmar que sea la única influencia, el profesorado constituye una parte importante en el desarrollo de ciertas actitudes en las mujeres.

Subirats (1994) indica que con frecuencia los docentes realizan diferenciación entre hombres y mujeres, señalando que ellas están "dotadas" para el lenguaje y ellos para las "matemáticas". Estos juicios de valor del cuerpo docente son influenciados por los estereotipos de género socialmente dominantes que también han interiorizado y que inconscientemente ayudan reproducir (Graña, 2006). Aún más, Jimeno (2002) señala que el profesorado, independientemente de su sexo, mantiene creencias diferentes para los hombres y las mujeres respecto a la educación 
matemática. Por ejemplo, se tiene la concepción de que los hombres se arriesgan más, son más independientes, disfrutan más con las matemáticas y se comprometen más en resolver problemas respecto a las mujeres.

Flores (2007), basada en datos recolectados a través de la observación, identifica algunos aspectos de las prácticas educativas de docentes de matemáticas que permitirían comprender algunas de las diferencias que se plantean en dicha disciplina respecto al género de los estudiantes. Algunos de los elementos observados son:

a) Existe una tendencia del profesorado al uso de un lenguaje docente masculinizado, lo que fortalece las diferencias entre géneros.

b) El profesorado tiende a dirigirse a los hombres y no a las mujeres durante la clase. Se estima que esto tiene implicaciones directas en la participación, especialmente en la frecuencia, manifestada por las mujeres en la clase mediante un menor número de intervenciones.

c) Respecto del número de preguntas realizadas por el profesorado al alumnado de la clase, la mayor cantidad de ellas estuvo dirigidas a los alumnos, mientras que el menor número correspondió a las alumnas.

d) Las mujeres, por recibir durante las sesiones de clase un menor número de preguntas que los hombres, no cuentan con los estímulos, el reconocimiento y la ayuda que merecen como estudiantes para corregir equivocaciones o aumentar su autoestima. Por lo tanto, sufren una deficiencia en su enseñanza que muchas veces culmina en una falta de confianza académica y de logros educativos.

e) Los hombres son más activos verbalmente en las clases de los profesores, mientras que las mujeres son menos activas con los profesores que con las profesoras.

Jimeno (2002) señala que distintas investigaciones muestran cómo las interacciones entre profesores o profesoras y sus estudiantes evidencian cómo los primeros tienden a estructurar las clases de matemáticas de tal forma que se favorece más el aprendizaje de los hombres que el de las mujeres.

Al respecto, Flores (2007) indica que, durante la última década, diferentes estudios han demostrado que los estereotipos de género transmitidos en la escuela afectan los resultados académicos de las mujeres, quienes muestran una baja autoestima en su aptitud para aprender matemáticas, lo cual puede ser explicado, en parte, por las representaciones de género de profesores y profesoras de matemática.

Perdomo (2009), por ejemplo, cita lo expuesto por el matemático británico Ernest, quien admitió que “(...) la imagen popular de las matemáticas es que es difícil, fría, abstracta, ultra racional, importante y fundamentalmente masculina” (p. 14) y señala que esta imagen, como generalización de un pensamiento "cotidiano y común", pudo convertirse en un obstáculo para las mujeres para expresar su interés por las matemáticas.

Por su parte, Brown y Josephs (2001) señalan que hombres y mujeres difieren en su autoconfianza matemática, lo que posiblemente se deba a la internalización de estereotipos sociales sobre las diferencias de género en matemáticas (los hombres son "buenos" en esta disciplina y las mujeres son "deficientes") o bien podrían deberse al hecho de que los estereotipos sociales y otros factores inhiben a las mujeres a tomar tantos cursos de matemáticas como a los hombres. 
Los resultados de diversas investigaciones en distintos países han traído como consecuencia la ejecución de algunas medidas que, sin embargo, parecen resultar insuficientes. Bayer (2004) menciona que aunque el número de mujeres que se doctoran en matemáticas y que después ejercen la docencia e investigación ha crecido, su presencia en centros de investigación internacionales, en conferencias plenarias y comités científicos de congresos y en comités editoriales de revistas de investigación es todavía reducida.

Por su parte, Jimeno (2002) señala que aunque ha aumentado considerablemente la presencia de las mujeres en carreras relacionadas con las matemáticas y que las diferencias entre los hombres y mujeres en esta disciplina han desaparecido en muchos países y en otros se han reducido considerablemente, su presencia en las carreras de mayor prestigio social sigue siendo menor. Para ampliar esta visión, en la siguiente sección se presentan los resultados de algunas investigaciones que evidencian la posición de desventaja que poseen las mujeres respeto a los hombres en matemáticas.

\section{Resultados de investigaciones sobre género y matemática}

Dado que diversas investigaciones han indicado diferencias entre hombres y mujeres respecto a las matemáticas, ha surgido la necesidad de buscar las causas de ello (Jimeno, 2002). En este proceso se han encontrado discrepancias sobre si son factores biológicos o ambientales, o una mezcla de ambos. Un gran número de investigaciones defienden la interacción de los factores ambientales y biológicos y, una buena parte de ellas, realizadas desde una perspectiva feminista, defienden que son causas ambientales.

Al respecto, Espinosa (2010) apunta que la mayor parte de estudios sobre género y matemáticas se han desarrollado en países de habla inglesa, como Estados Unidos, Inglaterra y Australia, mientras que en los de habla hispana el tema representa una línea de investigación relativamente nueva. La autora señala que estos estudios han aumentado el interés por indagar sobre las diferencias en el desempeño e intervención de mujeres y hombres en el aprendizaje de las matemáticas y la participación de ambos sexos en esta disciplina. Además, apunta que este tipo de investigación se ha identificado por seguir, principalmente, cinco líneas basadas en tres enfoques.

Las líneas de investigación son: educación matemática y género desde un análisis histórico; interacciones que se generan al interior de las aulas de matemáticas; relaciones familiares y escolares como "caminos" que condicionan y establecen la estancia de las mujeres y los varones en el área de las matemáticas; logro y desempeño en la resolución de problemas matemáticos (aritmética, geometría y cálculo); intervenciones pedagógicas y didácticas con el propósito de incrementar la participación de las mujeres en áreas donde las Matemáticas son importantes.

Los enfoques mencionados por Espinosa (2010) corresponden a: estudios en torno a las concepciones generales de las mujeres en matemáticas; estudios en torno a las concepciones del estudiantado sobre su aprendizaje y desempeño matemático; estudios en torno a las concepciones que el profesorado tiene de sus estudiantes sobre el aprendizaje y desempeño matemático.

En cuanto a ello, Jimeno (2002) señala que, de acuerdo con el informe PISA, los hombres manifiestan un mayor interés por las matemáticas en prácticamente todos los países respecto a las mujeres, además de expresar un mejor autoconcepto que ellas en dicha disciplina; también, apunta que gran parte de las investigaciones sobre género y matemáticas han resultado de estudios 
que han empleado una perspectiva tradicional de investigación en Ciencias Sociales, basada en la observación de conductas por medio de instrumentos de medida como los tests, los cuales se han centrado en medir las diferencias de género respecto a capacidades y habilidades, actitudes, entre otras. Con el fin de intervenir en ellos y lograr mejorar los resultados, el propósito ha sido determinar los aspectos en los cuales las mujeres han tenido desventajas en relación con los hombres.

Ante estas diferencias, unos mantienen que se trata de habilidades adquiridas debido a una educación con características diferentes para uno u otro sexo en la mayoría de las culturas a lo largo de muchos siglos; otros dan interpretaciones biológicas más profundas y pretenden establecer una diferencia innata en el cerebro que concede más facilidad para el desarrollo de unas actividades intelectuales a los hombres y de otras a las mujeres. Quizás, la realidad es que sea una mezcla de las dos cosas (...). (Jimeno, 2002, p. 284)

El autor anterior señala que la creencia de que las matemáticas son un dominio masculino influye en las actitudes de las mujeres hacia estas, en la percepción de sus capacidades para tener éxito en esta materia, y en sus elecciones de proseguir estudios en ella. Subirats (1994) señala que en todas las ciencias, incluidas las matemáticas y las ciencias naturales, se puede comprobar cómo el énfasis dado a la figura masculina ha impedido la comprensión de algunos fenómenos estudiados, pues no ha permitido elaborar una visión real de estos.

Las diferencias entre ambos géneros en matemáticas se abordan en dos planos. Uno se centra en las mujeres, en sus capacidades, habilidades, actitudes, creencias y expectativas. Todo ello se investiga comparando estos factores en los dos géneros. Otro plano se ocupa de factores externos, la mayoría factores educativos (creencias, actitudes y expectativas de los profesores sobre cuestiones de género, interacción en el aula, relaciones en el aula, perspectiva masculina en el curriculum y los libros de texto, etc.), aunque también se ha tratado influencias de la sociedad en general, de la familia y el grupo de iguales. Ambos planos están estrechamente relacionados, pues las actitudes, creencias, expectativas, interacciones, etc., están fuertemente influenciadas por la familia, el grupo de iguales y la sociedad en el sentido más amplio, por lo que es difícil tratar los dos aspectos por separado (...). (Jimeno, 2002, p. 280)

Diversos estudios han señalado que los hombres poseen mejores resultados que las mujeres en matemáticas (Olaz, 1997; Cervini, 2002; Jimeno, 2002; UNESCO², 2003; Cueto y Secada, 2004; Saunders y Winter, 2004; Winkler, 2004; Espinosa, 2004; Graña, 2006; Mella, 2006; Redondón y Navarro, 2007; Cabezas, 2009; Cervini, 2009; March, 2009; Palacios, 2009; Cervini y Dari, 2009; Inda-Caro et al., 2010).

Sin embargo, algunos estudios han mostrado que las diferencias en matemáticas entre hombres y mujeres se han venido reduciendo (Inda-Caro et al., 2010). Salomone (2007) indica que las mujeres todavía no han alcanzado el mismo nivel de rendimiento que los hombres en matemáticas y ciencias, especialmente en pruebas tipo test y en los niveles más avanzados.

Incluso, autoras como Mella (2006) apuntan que la diferencia entre hombres y mujeres en

Organización de las Naciones Unidas para la Educación, la Ciencia y la Cultura. 
los resultados en matemáticas resulta "discutible" desde todo punto de vista, y señala que basta con revisar algunas mediciones realizadas en países europeos, los cuales indican que la diferencias no son estadísticamente significativas o que, incluso, son las mujeres las que están obteniendo resultados mejores en matemáticas.

Al respecto, Jimeno (2002) señala que la mayoría de los estudios apuntan que parte de las diferencias en las tareas matemáticas que se presentan entre los hombres y las mujeres tienen relación con los procesos visoespaciales. Sin embargo, la autora también señala que aunque los déficits severos en el procesamiento visoespacial pueden conducir a dificultades de aprendizaje en matemáticas, no están tan claras las relaciones entre estos déficits y el aprendizaje de esta materia.

Cabezas (2009), basado en los resultados e información expuesta en los cuestionarios de la prueba del Sistema de Medición de la Calidad de la Educación de Chile del año 2003, señala que el efecto de tener un profesor del mismo sexo que el del alumno tiene un impacto positivo en el desempeño mostrado en las pruebas de matemáticas. Las diferencias observadas entre instituciones que reciben a estudiantes de un mismo sexo con respecto a los mixtos, indica que el efecto "positivo" de las interacciones de género docente-estudiante es mayor en condiciones donde existe un solo género, lo que podría ser señal de que este tipo de ambiente puede servir para reducir las brechas por género y el origen de las mismas. Sin embargo, Cabezas (2009) aclara que objetivo no es segregar a hombres y mujeres estudiantes y profesores con el fin de aumentar los desempeños, pues lo realmente importante es reconocer la existencia de los efectos sobre la coincidencia del estudiantado y profesorado y que estos no deberían ser significativos en la enseñanza, si es que se busca un sistema educativo libre de sesgos, a favor o en contra, de género.

Bethencour y Torres (1987) señalan que, de acuerdo con algunos resultados de investigación, hay una tendencia aparente según la cual la diferencia entre los resultados de hombres y mujeres en matemáticas dependen de la edad, pues la literatura generada de este tipo de investigaciones parece indicar el establecimiento tardío de la superioridad de los hombres, lo que representa un cambio de la tendencia anterior que señalaba que se iba intensificando con la edad. Además, indican que en la misma disciplina la diferencia entre hombres y mujeres parece depender del tipo de tarea matemática de la que se trate. En particular, basados en la investigación realizada por dichos autores con estudiantes de la educación general básica española, específicamente en la isla de Tenerife, los autores encontraron que los hombres son mejores que las mujeres resolviendo problemas aritméticos.

Betz y Hackett (1983), mencionados por Olaz (1997), estudiaron la utilidad de la autoeficacia aplicada al ámbito de las matemáticas y la elección de carreras en estudiantes universitarios. Bandura (1987), mencionado por Olaz (1997), señala que la autoeficacia ha sido definida como los juicios de las personas acerca de sus capacidades para alcanzar niveles determinados de rendimiento. Entre los principales hallazgos se encuentran que los estudiantes poseen creencias de autoeficacia más fuertes que las estudiantes y que la autoeficacia para las matemáticas está fuertemente relacionada con la toma de decisiones de carrera, lo cual muestra una importante relación entre las percepciones de autoeficacia para las matemáticas y la elección de carreras relacionadas con la ciencia.

Según Olaz (1997), los autores que sugieren estos resultados podrían ayudar a comprender el porqué del bajo porcentaje de mujeres en carreras universitarias relacionadas con las matemáticas y las ciencias. Sin embargo, el autor también apunta algunos estudios que señalan que las diferencias en matemáticas entre hombres y mujeres no son solamente por el sexo en sí, sino que responden a una construcción social. Indica que, desde la edad infantil, las mujeres y los hombres aprenden algunas 
diferencias asociadas a los sexos, entre las que destacan que los niños tienden a ser competitivos, aventureros, disfrutan de tareas que implican riesgos, gustan de construir cosas y de jugar con juguetes mecánicos, a la vez que están más interesados por tareas relacionadas con las matemáticas, las ciencias y la tecnología; mientras que a las niñas se les considera como cooperativas, cariñosas, gentiles, caritativas, intuitivas, más emotivas, menos racionales, y más interesadas por tareas como la lectura, la escritura, las danzas y las relaciones interpersonales, lo que podría ser, en parte, responsable de que sean pocas las que escojan carreras relacionadas con esta disciplina.

Graña (2008) señala que como parte de las conclusiones de una encuesta del Ministerio de Cultura y Educación en Buenos Aires, realizada a alumnos del último grado escolar de catorce establecimientos públicos y del último año del ciclo secundario de tres establecimientos privados, se obtuvo que las alumnas atribuyen las dificultades en matemáticas a su falta de capacidad personal en una proporción considerablemente mayor que los varones. También apunta que, basado en los datos de los 36 países para los cuales la UNESCO contaba con información estadística por sexo y por área desde 1985, los varones predominan en ingeniería; esta tendencia se verificaba igualmente en la mayoría de estos países para arquitectura, matemáticas, derecho y ciencias naturales.

Barbero, Holgado, Vila y Chacón (2007) realizaron un estudio de los datos del área de las matemáticas de la muestra española que participó en la segunda Evaluación Internacional del Progreso Educativo realizada por el Educational Testing Service. En la prueba que se les aplicó a dicha muestra, las preguntas relacionadas con matemáticas poseían cinco factores que representaban la capacidad de los estudiantes para resolver problemas relacionados con números y operaciones, geometría, análisis de datos, relaciones, funciones y expresiones algebraicas y cálculo. La muestra consistió de 2393 estudiantes de 13 años, donde 1188 eran hombres y 1205 eran mujeres.

Los resultados obtenidos reflejan las diferencias, según el género, en las respuestas. Los hombres poseen mejores resultados en geometría y cálculo, mientras que las mujeres se desempeñan mejor en análisis de datos y álgebra. Aunque no se presentaron diferencias entre los estudiantes según el sexo en cuanto a hábitos de estudio, sí hubo diferencia en su actitud hacia las matemáticas, donde fueron los hombres quienes presentaron una actitud más favorable hacia la disciplina.

Cabezas (2009) indica que las estadísticas disponibles para Chile señalan que los hombres obtienen mejores puntajes que las mujeres en las pruebas de matemáticas, mientras que ellas destacan en lenguaje. Estas diferencias son evidentes a medida que se avanza en la educación secundaria, pues los hombres aumentan la brecha entre matemáticas y lenguaje, con mejores resultados en la primera. Este tipo de comportamiento diferenciado por género persiste aún en educación superior (Cabezas, 2009; Jimeno, 2002).

González-Pienda et al. (2006), en una investigación realizada con estudiantes brasileños, tanto de primaria como de secundaria, encontraron que en ambas muestras las mujeres manifestaron una mayor desconfianza en sus logros en el área de las matemáticas, presentaron un mayor pensamiento estereotipado (pues argumentaron que las matemáticas son cosa de hombres) y una menor competencia percibida para el aprendizaje de las matemáticas.

March (2009), en un estudio realizado con estudiantes chilenos tanto de primaria como de secundaria, encontró que ambos sexos poseen un desempeño diferente a nivel escolar: se evidencia esta diferencia en matemáticas y lenguaje. Sin embargo, la autora señala que, al parecer, esta diferencia se "acorta" a través del tiempo. Además, señala que en matemáticas se observa que los hombres tienen un mejor desempeño con profesores hombres y las mujeres con profesoras mujeres: 
las mujeres superan en rendimiento, tanto en matemática como lenguaje a los hombres cuando se encuentran en colegios de solo mujeres. Los hombres, por su lado, se desempeñan mejor en escuelas mixtas en ambas materias, por lo que podría decirse que la mezcla de géneros favorece al aprendizaje de los hombres y desfavorece el de las mujeres. No obstante, esto amerita un estudio más acabado.

Pérez-Tyteca et al. (2009), basados en el estudio realizado con 885 estudiantes de la Universidad de Granada, provenientes de áreas como Ciencias de la Salud, Ciencias Experimentales, Ciencias Sociales y Enseñanzas Técnicas, con el propósito de estudiar la ansiedad matemática que presentan los alumnos recién ingresados en dicha universidad, hallaron que los hombres sufren menos ansiedad al enfrentarse a tareas matemáticas en comparación con las mujeres, situación que se presentó independientemente del área de estudio en la cual se encontraban.

Caprile et al. (2008) desarrollaron un estudio con una muestra de estudiantes de primaria de España, con el propósito de indagar aquellas dinámicas que se presentan en este ámbito escolar y que permitieran comprender por qué las mujeres eligen en menor medida que los hombres estudios científico-técnicos. Como parte los resultados encontrados se puede decir que, en relación con los libros de matemáticas, se observó que no se refleja un esfuerzo destacable por emplear un lenguaje no sexista, puesto que en la mayor parte de registros encontrados se utiliza el término masculino para referirse a personas de ambos sexos.

Espinosa (2004), con base en un estudio realizado con estudiantes de primaria de tres instituciones distintas de Lima, Perú, encontró, además de la diferencia respecto al rendimiento en matemáticas, que se presenta una mayor participación voluntaria de los hombres en el desarrollo de la clase de matemáticas.

Cervini y Dari (2009) analizaron los efectos del género en el logro del estudiantado en lengua y matemáticas del último año de la escuela secundaria en Argentina. Los investigadores hallaron que el género es un factor que afecta la distribución de los aprendizajes en matemáticas y en lengua de los estudiantes que se egresan de la educación secundaria. Señalan, además, que en promedio los puntajes obtenidos por las mujeres en lengua son notablemente superiores a los de los hombres, mientras que en matemáticas son ellos los que obtienen mejores resultados, pero no tan marcados como los de lengua.

Postigo et al. (1999) realizaron un estudio con estudiantes de educación primaria de España y encontraron que los hombres tienen una mayor confianza en su habilidad para resolver tareas matemáticas y científicas que sus compañeras. Al respecto, González (2005) señala que los estereotipos de género en matemáticas son uno de los factores que ayudan a explicar el desinterés por las matemáticas; en el caso especial de las mujeres, la forma de actuar del profesorado y los estereotipos de las matemáticas influyen en mayor medida en su desinterés en comparación con los hombres. La autora realizó un estudio con estudiantes de secundaria de México, con el propósito de validar un modelo teórico que busca explicar el desinterés por la matemática. Entre los resultados reportados por se puede mencionar que la tipificación de las matemáticas como dominio masculino es una variable que influye negativamente en el interés de los estudiantes, pues las mujeres que en mayor medida tipifican a las matemáticas como dominio masculino son las que poseen aprovechamiento más bajo.

Por su parte, Chavarría (1994b) realizó un estudio cuyo objetivo fue investigar si en los libros de texto de matemáticas que se utilizaban en Estados Unidos y Costa Rica se notaba diferencia en 
la distribución, debida al sexo, de los modelos ahí representados. En ambos países los resultados mostrados señalan que existe un mayor número de modelos masculinos a los cuales se hace referencia en los textos. En el caso de Costa Rica, las situaciones donde se presentan mujeres son muy diferentes a la de los hombres, por ejemplo, problemas donde las mujeres gastan dinero y los hombres lo ganan. Chavarría (1994b) señala, incluso, que se pudo observar situaciones abiertamente discriminatorias como el caso de salarios inferiores para las mujeres, deportes, peso y masa cerebral.

Incluso en Costa Rica, tanto a nivel de graduados de la Universidad de Costa Rica, única universidad que imparte la carrera de matemática pura y aplicada, como a nivel administrativo y docente relacionado con el área de matemáticas, se presentan diferencias.

Por ejemplo, Láscaris-Comneno et al. (2001) reportan que en Costa Rica, en el período 19901999, se graduaron un total de 49 personas en el área de las matemáticas, de los cuales solamente 3 correspondieron a mujeres. Además, señalan que, en ese mismo período, seis personas obtuvieron un posgrado en matemática en Costa Rica, todos hombres. Láscaris-Comneno et al. (2001) reportan otros datos interesantes como: en el periodo de 1993-1999 ninguna mujer ocupó la dirección de la Escuela de Matemática del Instituto Tecnológico de Costa Rica; en el periodo 1997-1999 ninguna mujer fungió como directora ni del Centro de Investigación en Matemáticas y Metamatemática (CIMM) ni del Centro de Investigación en Matemáticas Puras y Aplicadas (CIMPA) de la Universidad de Costa Rica; en el periodo 1990-1999 solamente en el año de 1990 una mujer ocupó la dirección de la Escuela de Matemática de la Universidad de Costa Rica; en el periodo 1990-1999 ninguna mujer ocupó la dirección de la Escuela de Matemática de la Universidad Nacional.

El Instituto de Estudios de la Mujer (IEM) de Universidad Nacional (2008) elaboró un documento donde, basado en datos cuantitativos, presenta las condiciones de inequidad entre hombres y mujeres en esta casa de estudios. Respecto al área de las matemáticas el estudio señala que la mayoría del personal docente de la Escuela de Matemática son hombres. Situación que se presenta en la otras "ciencias duras" como Física, Topografía, Economía, Biología, Química, Informática y Ciencias Agrarias. Incluso se menciona que en el Departamento de Física, Topografía, Matemática e Informática el número de mujeres académicas es mucho menor respecto a los hombres.

Estos datos muestran cómo en la enseñanza de la matemática y en la disciplina en general se presenta una relación de desigualdad entre hombres y mujeres. Factores hay muchos y los estudios anteriores señalan algunos de ellos. La responsabilidad de los docentes y las docenes es cambiar esta realidad y buscar acciones en beneficio de todo el estudiantado.

\section{¿Qué hacer?}

Perdomo (2009) señala que estudiosas en el tema de género y matemática defienden la necesidad de desarrollar una educación igualitaria enfocada en una transmisión de valores alternativos que no muestren a dicha disciplina como un conjunto de conocimientos "cerrado" y completo.

Al respecto, Ana Salvador, Adela Salvador y Molero (1991) indican que es importante hacer ver a la sociedad que no existe causa biológica que determine una diferencia de género, pues cualquier niña, al estar convencida de que una causa biológica es inalterable, se rendirá con facilidad a las dificultades. 
Erradicar los estereotipos de género por área de conocimiento, principalmente en ciencias y matemáticas, debería ser una política educativa en todos los niveles de la educación formal (González, 2005). Si las matemáticas se ven de una manera absolutista, inmutable, estática, sin posibilidad de cambio y universal tendría una visión pesimista para las mujeres; conocer las limitaciones, los sesgos y la estructura de las creencias de las matemáticas ayuda a determinar cómo debe cambiarse (Chavarría, 1994a).

Chavarría (1994b) señala que debiera existir una matemática "más femenina" que incluya los intereses y contemple la problemática de estas, construida mediante procesos que tomen en cuenta el contexto y más acordes con cómo las mujeres aprenden. Al respecto, Ayala (2009) apunta que atender el género en la enseñanza de las matemáticas se refiere a considerar que las mujeres en este campo tienen las mismas potencialidades que los hombres y las mismas exigencias.

Las diferencias de género en los resultados educativos eran casi universalmente a favor de los hombres, pero se han ido estrechando, lo que parece indicar que algunos países han cambiado para establecer un sistema educativo que beneficie por igual a ambos géneros, muchos de ellos como resultado de esfuerzos educativos o por un contexto social más favorable (Jimeno, 2002). En cuanto a ello, Jimeno (2002) indica que las investigaciones realizadas en el aula han puesto de manifiesto que las mujeres aprenden mejor en ambientes donde existe un clima de cooperación y no se alienta la competitividad.

A pesar de que en las últimas décadas la preocupación por la equidad ha ido en aumento, la corriente principal de investigaciones sobre educación matemática no tiene muy en cuenta las cuestiones sobre equidad; se centra, en su mayoría, en los aspectos psicológicos y la propia disciplina, olvidando los aspectos sociales. Se da prioridad a los aspectos individuales del aprendizaje, a las investigaciones sobre cómo aprenden y a la estructura y contenidos de la disciplina y se olvida que el aprendizaje de las matemáticas en la escuela es un acto social, cultural y político. Que las matemáticas, lejos de estar libres de valores, ser apolíticas y alejadas del mundo social, juegan un papel crucial en el desarrollo de nuestras sociedades y democracias y en el papel en la sociedad de nuestros estudiantes. Promover la equidad requiere reconocer que las matemáticas no están libres de valores, no son neutrales culturalmente, ni imparciales políticamente (...). (Jimeno, 2002, p. 370)

En este sentido, la autora señala que la equidad en matemáticas implica cambios profundos en los sistemas educativos y en las escuelas, en instituciones y en una sociedad realmente comprometida. Por ello indica que las aulas de matemáticas deberían ser lugares donde:

a) Todos y cada uno de los componentes del aula tengan el derecho a hablar y a que se les muestre el respeto que merecen sus ideas y sus palabras.

b) Los conflictos que puedan surgir se resuelvan entre todos, como en una comunidad democrática en la que reina la cooperación.

c) No se fomenta la discriminación ni a través del lenguaje escrito u oral o de cualquier otro tipo de conductas, sino al contrario, se lucha contra ella.

Y las matemáticas escolares deberían partir de conocimientos que concuerden con la vida de los estudiantes y las estudiantes, no de conocimientos estereotipados que reflejen experiencias únicas; 
contemplar la rica variedad de experiencias que puede aportar cada estudiante del aula; valorar la intuición y la emoción, así como las diferentes formas de abordar y resolver las tareas académicas; evitar la desvalorización de las formas más prácticas y concretas de resolución de tareas o situaciones; fomentar un pensamiento crítico y reflexivo. En particular, es importante que los niños y niñas sean conscientes y reflexionen sobre sus propios procesos de pensamiento y modos de resolución de problemas.

Salvador et al. (1991) señalan que en una educación igualitaria, donde a las mujeres se les exima de las tareas domésticas y del cuidado de los hermanos pequeños y con las mismas expectativas familiares respecto a los hombres, no existiría diferencia entre ellos y ellas en relación con su capacidad para las matemáticas. El colaborar para que se eduque desde la diferencia y para esta es una tarea de todos los involucrados y todas la involucradas en el proceso educativo, y no solo de algunos cuantos. Debe ser una prioridad nacional donde se inicie por concientizar, tanto al personal docente como a estudiantes y padres y madres de familia, de que las relaciones de desigualdad se presentan. Como docentes de matemáticas debemos tomar conciencia de que esa realización de la persona en todos los aspectos, postulada por los fines de la enseñanza de las matemáticas, está quedando incompleta y debemos llevar a cabo acciones tendientes a corregir dicha situación.

\section{Conclusiones}

La inequidad en la enseñanza de las matemáticas es una realidad que abarca diversos aspectos. Distintas investigaciones han señalado que el sistema educativo está estructurado en torno a lo considerado importante: el hombre ha sido uno de estos elementos. Las matemáticas se han concebido como un conocimiento fundamental para el ser humano, por lo que se destaca su importancia. Por lo tanto, la asociación matemática-masculino ha tenido un papel preponderante, en perjuicio de las mujeres, en la enseñanza de esta disciplina.

Al estudiantado se le "ha vendido la idea" de que hombres y mujeres son diferentes en su capacidad para desempeñarse en dicha disciplina y la mayor parte del proceso educativo se ha estructurado en torno a dicha idea.

Pero, ¿quién le enseña a la niña y al niño estas conductas? La influencia de los padres y madres de familia, el profesorado y el medio social en la construcción de la "identidad matemática" es innegable. Las diferencias de género en la enseñanza de las matemáticas están influenciadas fuertemente por los estereotipos que el estudiantado ha interiorizado y que lo "determina" a un comportamiento establecido.

La enseñanza de la disciplina no hace nada, o por lo menos muy poco, para "decodificar" dichas conductas. Por el contrario, muchos de los elementos que intervienen en ella contribuyen a afianzar las diferencias. Creo que la situación no cambiará si antes no se realiza un proceso de concientización del profesorado. Inconscientemente muchas de las profesoras y los profesores de secundaria contribuyen a perpetuar la desigualdad y "transmisión" de estereotipos. Ayudarlos y ayudarlas a corregir esa tarea es un compromiso de todos y todas.

Por lo tanto, es necesario que, de forma conjunta, se lleven a cabo acciones que permitan modificar la "identidad matemática" del estudiantado y su percepción de aprendiz ante dicha disciplina, se incentive el ingreso equitativo de hombres y de mujeres a las distintas carreras que han sido tipificadas como predominantemente femeninas o masculinas y se permita, tanto a hombres como a mujeres, valorar las diferencias para construir relaciones fundamentadas en el respecto, la solidaridad y la ayuda mutua. 


\section{Referencias}

Ayala, A. (mayo, 2009). Currículo y educación matemática. Tarea, 71, 18-21. Recuperado de http:// tarea.org.pe/modulos/Boletin/revistas/Tarea_71/Tarea\%2071\%20_\%2018\%20Ana\%20Ayala. pdf

Barbero, M. I., Holgado, F. P., Vila, E. y Chacón, S. (2007). Actitudes, hábitos de estudio y rendimiento en matemáticas: diferencias por género. Psicothema, 19(3), 413-421. Recuperado de http://redalyc.uaemex.mx/src/inicio/ArtPdfRed.jsp?iCve=72719309

Bayer, P. (enero-abril, 2004). Mujeres y Matemáticas. La Gaceta de la Real Sociedad Matemática Española, 7(1), 55-71. Recuperado de http://gaceta.rsme.es/vernumero.php?id=47

Bethencour, J. T. y Torres, E. (1987). La diferencia de sexo en la resolución de problemas aritméticos: un estudio transversal. Inteligencia y aprendizaje, 38, 9-20. Recuperado de http://dialnet. unirioja.es/servlet/articulo?codigo $=662325$

Brown, R. y Josephs, R. (abril, 2001). El peso de la prueba: Diferencias de género y relevancia de los estereotipos en el desempeño matemático. Nómadas, 14, 110-123. Recuperado de http:// redalyc.uaemex.mx/redalyc/pdf/1051/Resumenes/105115268010 Resumen 1.pdf

Cabezas, G. (diciembre, 2009). ¿Afecta el género de los profesores los resultados académicos de los estudiantes? Diferencias de género en matemática y lenguaje (Documento de Trabajo CPCE $\mathrm{N}^{\circ}$ 15). Chile: Centro de Políticas Comparadas de Educación. Recuperado de http://www.cpce. cl/publicaciones/documentos-de-trabajo/121-15-iafecta-el-genero-de-los-profesores-a-losresultados-academicos-de-los-estudiantes-diferencias-de-genero-en-matematica-y-lenguaje

Caprile, M. (Coord.), Vallès, N., Potrony, J., Crèixams, C. y Arasanz, J. (octubre, 2008). El sesgo de género en el sistema educativo. Su repercusión en las áreas de Matemáticas y Tecnología en secundaria (THEANO). Ministerio de Igualdad. España. Instituto de la Mujer. Fundación CIREM.

Cervini, R. (2002). Desigualdades socioculturales en el aprendizaje de Matemática y Lengua de la educación secundaria en Argentina: Un modelo de tres niveles. Revista Electrónica de Investigación y Evaluación Educativa RELIEVE, 8(2), 135-158. Recuperado de http://www. uv.es/RELIEVE/v8n1/RELIEVEv8n2 $1 . \mathrm{htm}$

Cervini, R. (2009). Comparando la inequidad en los logros escolares de la educación primaria y secundaria en Argentina: Un estudio multinivel. REICE. Revista Iberoamericana sobre calidad, eficacia y cambio en educación, 7(1), 5-21. Recuperado de http://www.rinace.net/ reice/numeros/arts/vol7num1/Reice,\%207(1).pdf\#page $=8$ 
Cervini, R. y Dari, N. (octubre-diciembre, 2009). Género, escuela y logro escolar en matemática y lengua de la educación media: Estudio exploratorio basado en un modelo multinivel bivariado. Revista Mexicana de Investigación Educativa, 14(43), 1051-1078. Recuperado de http://www. comie.org.mx/v1/revista/portal.php?idm $=$ es $\& s e c=S C 03 \& \&$ sub $=S B B \&$ criterio=ART43004

Chavarría, S. (setiembre, 1994a). Matemática sesgada por género. Revista de Ciencias Sociales, 65, 127-132.

Chavarría, S. (junio, 1994b). Ocupaciones y sesgo de género es los libros de texto de matemática de secundaria. Revista Casa de la Mujer, 4(5), 24-32.

Colclough, C. (2003). Educación para todos: Hacia una igualdad entre los sexos. [Resumen del informe de seguimiento de la Educación para todos en el mundo 2003/4]. París, Francia: Ediciones UNESCO. Recuperado de http://www.unesco.org/education/efa_report/2003_pdf/ summary es.pdf

Cueto, S. y Secada, W. (2004). Oportunidades de aprendizaje y rendimiento en matemática de niños y niñas Aimara, Quechua y Castellano hablantes en escuelas bilingüies y monolingües en Puno, Perú. En D. Winkler y S. Cueto (Eds.). Etnicidad, raza, género y educación en América Latina (pp. 315-353). Washington: Programa de promoción de la reforma educativa en América Latina y el Caribe.

Espinosa, C. (marzo, 2010). Diferencias entre hombres y mujeres en educación matemática: ¿Qué pasa en México? Investigación y Ciencia, 18(46), 28-35.

Espinosa, G. (2004). Currículo y equidad de género en la primaria: Una mirada desde el aula. Estudio realizado en tres escuelas estatales de la ciudad Lima. En M. Benavides (Ed.), Educación procesos pedagógicos y equidad. Cuatro informes de investigación, 69-129. Lima, Perú. Recuperado de http://www.grade.org.pe/download/pubs/libros/EPPyEquidad.pdf

Flores, R. (enero-abril, 2007). Representaciones de género de profesores y profesoras de matemática, y su incidencia en los resultados académicos de alumnos y alumnas. Revista Iberoamericana de Educación, 43, 103-118.

González, R. M. (agosto, 2003). Diferencias de género en el desempeño matemático de estudiantes de secundaria. Educación Matemática, 15(2), 129-161.

González, R. M. (abril, 2005). Un modelo explicativo del interés hacia las matemáticas de las y los estudiantes de secundaria. Educación Matemática, 17(1), 107-128.

González-Pienda, J. A., Núñez, J. C., Álvarez, L., González, P., González-Pumariega, S., Roces, C., Castejón, L., Solano, P., ... Socorro, L. (2006). Diferencias de género en actitudes hacia las matemáticas ( $2^{\mathrm{a}}$ ed.). Braga: Universidade do Minho. 
Graña, F. (diciembre 2006). Una revisión de estudios recientes: Igualdad formal y sexismo real en la escuela mixta. Revista de Ciencias Sociales, Año XIX, 23, 63-75.

Graña, F. (2008). El asalto de las mujeres a las carreras universitarias "masculinas": Cambio y continuidad en la discriminación de género. PRAXIS educativa, 12, 77-86. Recuperado de http://redalyc.uaemex.mx/src/inicio/IndArtRev.jsp?iCveNumRev=12902\&iCveEntRev=1531\#

Inda-Caro, M., Rodríguez-Menéndez, C. \& Peña-Calvo, V. (2010). PISA 2006: La influencia del género en los conocimientos y competencias científicas. Revista Iberoamericana de Educación, 51(2), 1-12.

Instituto de Estudios de la Mujer, Universidad Nacional. (2008). Diagnóstico institucional. Relaciones de equidad entre mujeres y hombres en la Universidad Nacional. Heredia, Costa Rica: Facultad de Filosofía y Letras, Universidad Nacional.

Jimeno, M. (2002). Al otro lado de las fronteras de las matemáticas escolares: Problemas y dificultades en el aprendizaje matemático de los niños y niñas de tercer ciclo de primaria. (Tesis doctoral, Departamento de Didáctica y Organización Escolar. Facultad de Ciencias de la Educación. Universidad de Málaga, España). Recuperado de riuma.uma.es/xmlui/bitstream/ handle/10630/2552/16275718.pdf?...

Láscaris-Comneno, T, Aguilar, G., Silva, S., Coto, J. M., Calderón, O. y Brenes, I. (2001). Ciencia, tecnología y género en Costa Rica 1990-1999. San José, Costa Rica: UNESCO, OEI., Proyecto Iberoamericano de Ciencia, Tecnología y Género. Recuperado de www.oei.es/ $\underline{\text { salactsi/tatiana.pdf }}$

March, D. A. (2009). Diferencias de género en rendimiento académico: Efectos en la evaluación a nivel escolar (Memoria). Escuela de Ingeniería, Pontificia Universidad Católica de Chile, Santiago, Chile. Recuperado de http://www.ideaseneducacion.cl/wp-content/uploads/2009/06/ memoria-daniela-march.pdf

Mella, O. (2006). Factores que afectan los resultados de la escuela pública chilena. Revista Electrónica Iberoamericana sobre Calidad, Eficacia y Cambio en Educación (REICE), 4(1), 29-37. Recuperado de http://redalyc.uaemex.mx/pdf/551/55140104.pdf

Olaz, F. (1997). Autoeficacia, diferencias de género y comportamiento vocacional. Revista electrónica de Motivación y Emoción, 6(13). Recuperado de http://reme.uji.es/articulos/ aolazf5731104103/texto.html

Palacios, L. (2009). Epistemología y pedagogía de género: El referente masculino como modo de construcción y transmisión del conocimiento científico. Horizontes Educacionales, 14(1), 65-75. Recuperado de http://redalyc.uaemex.mx/src/inicio/ArtPdfRed.jsp?iCve=97912444005 
Perdomo, I. (2009). Matemática y género. Una aproximación histórica. Organización de Estados Iberoamericanos (OEI), 1-30. Recuperado de http://www.oei.es/salactsi/ MatematicasyGeneroUnaaproximacionhistorica1.pdf

Pérez-Tyteca, P., Castro, E., Segovia, I., Castro, E., Fernández, F. y Cano, F. (2009). El papel de la ansiedad matemática en el paso de la educación secundaria a la educación universitaria. PNA, 4(1), 23-35.

Postigo, Y., Pérez, M. y Sanz, Á. (1999). Un estudio acerca de las diferencias de género en la resolución de problemas científicos. Enseñanza de las Ciencias, 17(2), 247-258. Recuperado de http://dialnet.unirioja.es/servlet/articulo?codigo $=94958$

Redondón, S. y Navarro, E. (2007). Estudio sobre el rendimiento en matemáticas en España a partir de los datos del informe PISA 2003. Un modelo jerárquico de dos niveles. Revista Electrónica Iberoamericana sobre calidad, Eeficacia y cambio en Educación (REICE), 5(3), 118-136. Recuperado de http://www.rinace.net/arts/vol5num3/art13.pdf

Salomone, R. (2007, septiembre-diciembre). Igualdad y diferencia. La cuestión de la equidad de género en la educación. Revista española de pedagogía, 65(238), 433-446.

Salvador, Ana; Salvador, Adela y Molero, M. (septiembre, 1991). Mujeres y matemáticas: Un estudio diferencial. Números, 21, 57-65. Recuperado de http://www.sinewton.org/numeros/ numeros/21/Articulo06.pdf

Saunders, M. y Winter, C. (2004). Las desigualdades de género en la educación en América Latina: Resultados de las nuevas investigaciones. En D. Winkler y S. Cueto (Eds.) Etnicidad, raza, género y educación en América Latina (pp. 33-53). Perú: PREAL.

Subirats, M. (1994, septiembre-diciembre). Conquistar la igualdad: la coeducación hoy. Revista Iberoamericana de Educación, 6, 49-78. Recuperado de http://www.rieoei.org/oeivirt/rie06. $\underline{\mathrm{htm}}$

Susi, E. (1994). Ciencia y género: Autoridad y medida en la enseñanza. Enseñanza de las Ciencias, 12(2), 200-205.

Toríz, A. (2004, diciembre). Reseña de "Género y matemáticas: balanceando la ecuación” de Rosa María González. Educación Matemática, 16(3), 163-166.

Winkler, D. (2004). Investigaciones sobre etnicidad, raza, género y educación en las américas. En D. Winkler y S. Cueto (Eds.) Etnicidad, raza, género y educación en América Latina (pp. 1-32). Perú: PREAL. 